\title{
A rare case of mediastinal fibromatosis in a child - case report
}

\author{
Dorota Aleksandra Nadbrzeżna' ${ }^{1}$, Aleksandra Raźnikiewicz², Andrzej Grabowski², \\ Wojciech Korlacki², Tomasz Szczepański ${ }^{3}$, Joanna Bulsa ${ }^{3}$, Małgorzata Walaszczyk ${ }^{4}$ \\ 'Department of General Surgery, Municipal Hospital Complex in Chorzow, Poland \\ 2Department of Children's Developmental Defects Surgery and Traumatology, School of Medicine with the Division \\ of Dentistry in Zabrze, Medical University of Silesia in Katowice, Zabrze, Poland \\ ${ }^{3}$ Department of Paediatric Haematology and Oncology, School of Medicine with the Division of Dentistry in Zabrze, \\ Medical University of Silesia in Katowice, Zabrze, Poland \\ ${ }^{4}$ Department of Anaesthesiology and Intensive Therapy, School of Medicine with the Division of Dentistry in Zabrze, \\ Medical University of Silesia in Katowice, Zabrze, Poland
}

\section{ABSTRACT}

Introduction: Fibromatosis is a very rare desmoid tumour. It is characterised by local invasion and infiltration of close structures. The treatment is mainly surgical.

Material and methods: We report the case of 1.5-year-old boy admitted to the Paediatric Hospital, with a diagnosis of mediastinal desmoid fibromatosis. He was treated surgically (thoracoscopy and thoracotomy), chemotherapy was used, and he was hospitalised in the Intensive Care Unit more than once. Despite intensive treatment, the patient perished.

Results and conclusions: Fibromatosis is classified as a desmoid tumour. Rare tumour localisation and invasive tumour characteristics causes considerable difficulty in the diagnosis, despite the use of many diagnostic methods.

\section{KEY WORDS:}

thoracotomy, mediastinum, fibromatosis, desmoid tumour.

\section{INTRODUCTION}

Desmoid tumour (DT), also known as aggressive fibromatosis, is defined by the World Health Organisation as "a clonal fibroblastic proliferation that arises in the deep soft tissues and is characterised by infiltrative growth and a tendency toward local recurrence but an inability to metastasise" [1]. The first DT description was made by MacFarlane in 1832 [2].

DF accounts for $0.03 \%$ of all neoplasms and $3 \%$ of soft tissue tumours [3]. Thus, the incidence of DF is five to six cases per year per 1 million of the population [4]. DF mainly affects people between 10 and 40 years of age and is more common in the female population $[1,5]$. The main method of treatment is surgery, but radiotherapy, chemotherapy, hormonotherapy, immunotherapy, and nonsteroidal anti-inflammatory drugs are also used [6, 7]. The prognosis is uncertain, and the mortality rate varies from $0 \%$ to $8 \%$.

\section{CASE REPORT}

A 12-month-old boy with a history of refractory bronchospasm was admitted to the Department of Paediatric Haematology and Oncology due to massive tumour of the chest.

\section{ADDRESS FOR CORRESPONDENCE:}

Andrzej Grabowski, Department of Children's Developmental Defects Surgery and Traumatology, School of Medicine with the Division of Dentistry in Zabrze, Medical University of Silesia in Katowice, 13-15 3 Maja St., 41-800 Zabrze, Poland, ORCID: 0000-0002-5162-3043, e-mail: agrabowski@szpital.zabrze.pl 
His history revealed asymmetric swelling of the left lower limb and gluteal region, presented at birth, which was referred with additional tests including X-ray, computed tomography (CT), and magnetic resonance imaging (MRI). Detailed investigation revealed benign hypertrophy of fatty and fibrotic tissue of the left lower limb. The additional genetic diagnostics was inconclusive.

At admission the patient presented breathing difficulties, wheezing and dyspnoea. There was no evidence of infection. The chest X-ray revealed a massive tumour of the anterior mediastinum (Fig. 1). Chest-enhanced CT after admission demonstrated (Figs. 2 and 3) large, space-modelling right chest and mediastinal lesion $(115 \mathrm{~mm} \times 76 \mathrm{~mm} \times 97 \mathrm{~mm})$ with heterogenic architecture, necrotic area, and no evidence of calcification. The tumour arose from the anterior chest wall, causing displacement of the mediastinal structures laterally into the left side with vascular occlusion and narrowing the breathing airways and following atelectasis.

All laboratory findings were within normal values, and detailed staging investigation did not identify any signs of metastatic disease. After preoperative preparation with bronchodilators and steroids, thoracoscopy with open biopsy of the tumour was performed. Intraoperative exploration revealed a massive cauliflower-like shaped tumour with invasive growth into the right chest and mediastinum with infiltration of the sternum and chest wall. The operation was complicated by cardio-respiratory failure resulting in mechanical ventilation and further supportive treatment in the Intensive Care Unit.

Histologically, the tumour was composed of spindle cell proliferation. Immunohistochemical staining showed positive reaction for beta-catenin, vimentin, calponin and with foci positivity for smooth muscle actin (SMA) and C-kit, negative reaction for S-100 protein, desmin, and oestrogen receptor. These findings, confirmed by the reference pathologist, led to a final diagnosis of desmoid fibromatosis.

Taking into account highly progressive proliferation and incomplete resection of the mass, the treatment strategy scheduled chemotherapy according to the current treatment protocol CWS-2006 based on three subsequent VAC courses (vincristine, actinomycin-D, cyclophosphamide), following a further decision about the type of local treatment (complete resection vs. radiotherapy). A three-month follow-up MRI of the chest showed $20 \%$ progression in tumour size. Thus, the treatment CWS-2006 plan assumed a further three $\mathrm{I}^{3} \mathrm{VA}$ courses (ifosfamide, vincristine, acinomycin-D). However, after eight days of chemotherapy the patient demonstrated breathing problems, dyspnoea, and progressive respiratory failure. CT of the chest confirmed a significant increase in the size of the fibromatosis mass despite chemotherapy. Due to life-threating symptoms, the patient underwent emergency surgical intervention aimed at tumour reduction. A right posterolateral thoracotomy revealed

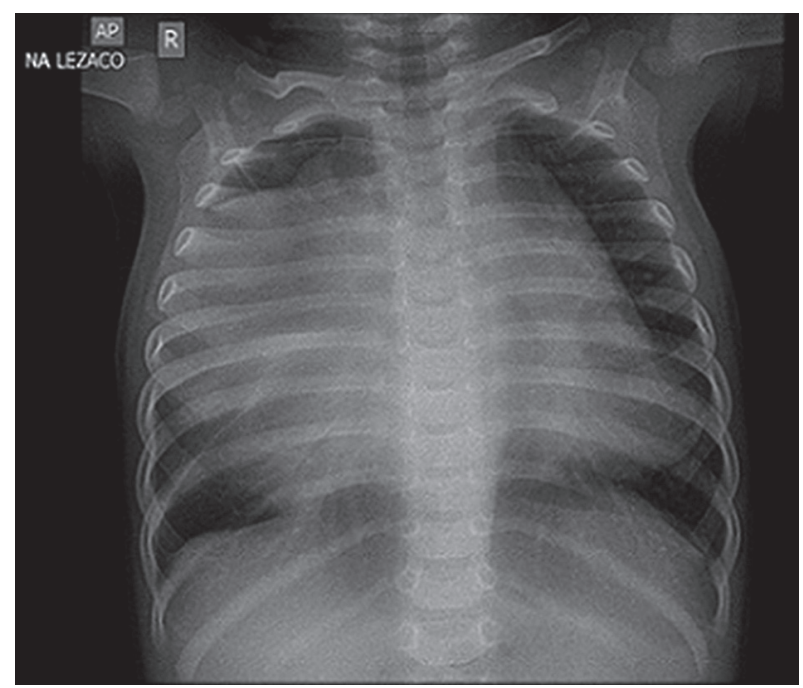

FIGURE 1. Chest X-ray: a large dilatation of the anterior mediastinum

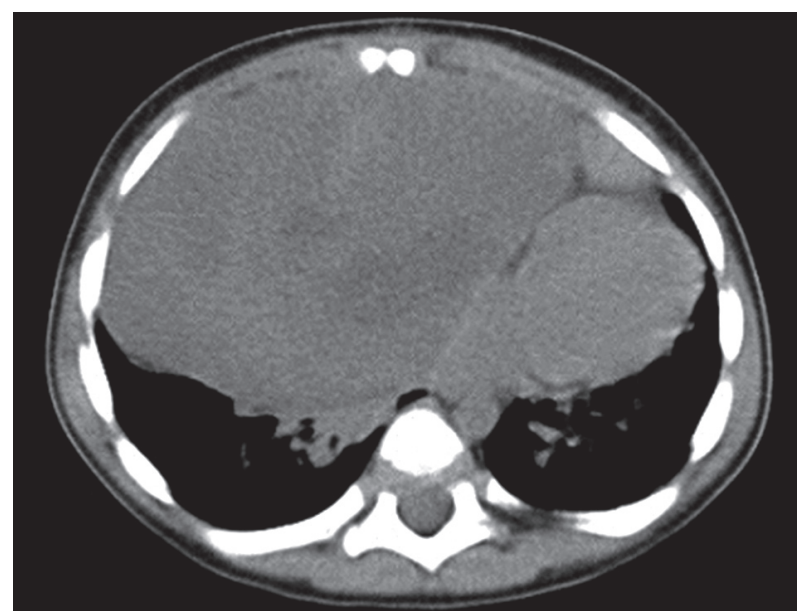

FIGURE 2. Computed tomography scan of the chest: tumour models the mediastinal structures

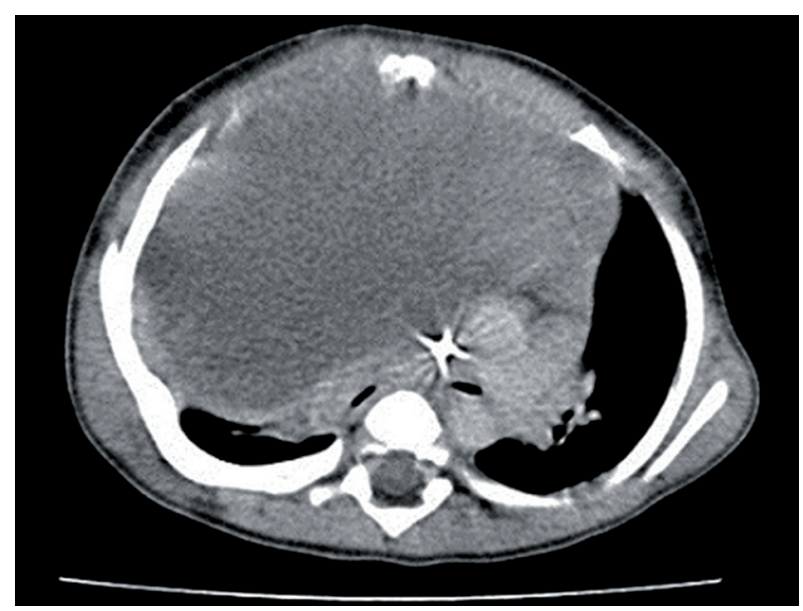

FIGURE 3. Computed tomography scan of the chest: the tumour at the chest wall in front of the previous scans significantly increased dimensions to about $11 \mathrm{~cm} \times 13 \mathrm{~cm} \times 10 \mathrm{~cm}$

a massive tumour of the right chest and mediastinum with infiltration into the trachea, pericardium, mediastinal vessels, and sternum. The mass was excised subtotally. There was no possibility for complete resection of the 
invasive mass adjacent to mediastinal organs. As a result of respiratory failure, extensive surgery, and the following critical condition, the patient required supportive treatment in an Intensive Care Unit. The major complications included respiratory failure, haemorrhagic shock and asphyxia. After 30 days of intensive therapy, the patient was readmitted to the Haemato-oncology Department for treatment continuation. The general condition was serious: the patient demonstrated oxygen-depended dyspnoea with tachypnoea and wheezing, neurological disturbances characterised by general slenderness, strabismus, and quantitative consciousness disorder. Moreover, as a complication of critical illness and related therapy, hepatosplenomegaly occurred.

Taking into account the serious state of the patient, no possibility for complete tumour resection, and high resistance to previous chemotherapy, the strategy of further treatment was extremely problematic. According to the multidisciplinary expert recommendations, the subsequent approach to this highly aggressive intra-thoracic lesion was started with combination therapy with an anti-hormonal agent (tamoxifen) and a non-steroidal anti-inflammatory drug (diclofenac) followed by monotherapy with a tyrosine kinase inhibitor. If progression was evident, chemotherapy with vinblastin and methotrexate (VBL/MTX) would have been started. The radiotherapy and further surgery were reserved for situations that were palliative, life-threating, or resistant to medical treatment. After initial stabilisation of the tumour mass with tamoxifen and diclofenac treatment, rapid progression occurred. Treatment with chemotherapy was initiated without any further result. Afterwards, due to resistance to VBL/ MTX, treatment with imatinib was started. The response to this management was also unsatisfactory. As a result of massive progression corresponding to respiratory failure, despite mechanical ventilation, eight months after first diagnosis the patient died.

\section{DISCUSSION}

In this report, we present a rare paediatric case of a rapidly growing DT of the chest with fatal course. Desmoid-type fibromatoses of the deep tissue are rare soft tissue tumours characterised by proliferation of fibroblast and myofibroblast-type spindle cells, which infiltrate musculoaponeurotic tissue [8]. The aetiology of DT remains unknown [9]. It is thought to be associated with trauma, surgery, and genetic predisposition (familial adenomatous polyposis, Gardner syndrome). The good response to anti-oestrogen therapy indicates that endocrine factors play an important role in tumour development [10-12].

The most common location are the extremities, followed by the abdomen, chest, then head and neck [13]. Tumours of the chest wall account for 10 to $20 \%$ of cases of DT, and intrathoracic locations are rare $[8,14]$. The symptomatology remains non-specific, mimicking normal childhood illnesses, and is common for any other type of thoracic tumour. The characteristic symptoms and signs are usually associated with mass effect and include dyspnoea, chest pain, chest deformation, cough, hoarseness, SOB without any evidence of infection and asthma, vena cava, and Horner's syndrome [15]. One of the most serious results of tumour expansion is occlusion of airways and main vessels, as well as infiltration of important organs including lungs and heart. DT should be distinguished from other paediatric tumours such as lymphomas, Langerhans histiocytosis, germ cell tumours, and other soft tissues lesions including rhabdomyosarcoma, fibrosarcoma, and PNET [16].

Both enhanced computed tomography and MRI are very important tools for early assessment of the tumour mass. Imaging is a mandatory examination for diagnosis of fibromatosis tumour, but it is non-specific. Despite variable images, MRI and CT are very helpful not only in evaluation many important tumour characteristics such as size, location, and margins, but also in staging assessment including anatomical association with other organs and lymph node involvement [5]. Thus, it is a valuable method for building optimal treatment strategy, surgery approach, and monitoring the response to applied therapy. An open biopsy of the tumour is necessary for diagnosis. Histopathological and immunohistochemical findings are usually the basic elements for final confirmation of the recognition [17].

According to current treatment protocols, the optimal management for DT remains controversial because the biologic behaviour of the tumour is unpredictable. The following have been reported: spontaneous regression, infiltrative and progressive rapid growth, and high incidence of local relapse after surgery $[18,19]$. The treatment approach should be based on assessment of local aggressiveness, growth potential, life-threating location, and influence on organ function [13].

Full surgical excision is the preferred treatment for symptomatic DT. While surgical resection remains the primary treatment for these tumours, published recurrence rates in the paediatric population range from $23 \%$ to $83 \%$ [20]. It is very important to assess whether the tumour was completely resected. Adjuvant therapy (systemic chemotherapy or radiotherapy) is reserved only for non-resectable aggressive fibromatosis [19].

Chemotherapy is an appropriate choice for patients who have rapidly growing or unresectable tumours or who are symptomatic [21]. Drugs used in children include methotrexate, vinblastine, vinorelbine, doxorubicin, dacarbazine, vincristine, actinomycin, etoposide, cyclophosphamide, and ifosfamide [13, 21].

According to current data, in the case of resistance to chemotherapy, alternative treatment has been proposed. The goal of this therapy is reduction of the tumour mass, to allow subsequent complete resection. The alternative 
strategy should be scheduled individually, and it mostly concerns experimental treatment in paediatric cases. The potential therapy includes hormonal agents, tyrosine kinase inhibitors, anti-inflammatory agents, and biological treatment [18].

The treatment strategy in the presented case required a multidisciplinary approach and individualised treatment decisions. Complete resection of this highly aggressive, extremely large DT, with a life-threating location was not possible. Despite surgical debulking, the chemotherapy with a combination of alternative treatment was not effective in preventing the patient from local recurrence and subsequent rapid progression. The stabilisation of tumour growth was only temporary, and further progressive occlusion of the airways and main mediastinal organs, resulting in increasing respiratory failure, was the direct cause of the patient's death.

\section{CONCLUSIONS}

The rareness of fibroma occurrence inside the chest and non-specific clinical symptoms causes considerable difficulty in the diagnosis, despite the use of many diagnostic methods. Even though these tumours do not metastasise, they can result in death from locoregional invasion and significant morbidity. As in our case, full surgical excision is often difficult to obtain; healthy margins due to the local anatomic conditions or the infiltrating nature of the lesions are hard to gain.

\section{DISCLOSURE}

The authors declare no conflict of interest.

\section{REFERENCES}

1. Fletcher CDM, Unni KK, Mertens F. Pathology and genetics of tumours of soft tissue and bone. World Health Organization classification of tumours, International Agency for Research on Cancer. IARC Press, Lyon 2013: 81-82.

2. Laufer I, Wolinsky J-P, Gokaslan ZL. Desmoid tumours. World Neurosurg 2013; 79: 97-98.

3. Fletcher JA, Bridge JA, Hogendoorn PCW, et al. WHO Classification of Tumours of Soft Tissue and Bone. $4^{\text {th }}$ ed. IARC Press, Lyon 2013: 72-73.

4. Penel N, Coindre JM, Bonvalot S, et al. Management of desmoid tumours: a nationwide survey of labelled reference centre networks in France. Eur J Cancer 2016; 58: 90-96.

5. Wilhelm A, Jolles HI, Krishna M. Anterior mediastinal desmoid tumour with CT and MR imaging. J Thorac Imaging 2007; 22: 252-255.

6. Reiter M, Schwope R. Intrathoracic desmoid type fibromatosis: a case report and literature review. J Surg Radiol 2012; 3: 184-188.

7. Sze H, Yeung MW. Fibromatosis of the neck causing airway obstruction managed effectively with weekly low-dose methotrexate and vinblastine. Hong Kong Med J 2009; 15: 221-223.

8. Ibrahim M, Sandogji H, Allam A. Huge intrathoracic desmoid tumor. Ann Thorac Med 2009; 4: 146-148.
9. Hosalkar HS, Fox EJ, Delaney T, et al. Desmoid tumors and current status of management. Orthop Clin North Am 2006; 37: 53-63.

10. Ayala AG Ro JY, Goepfert H, et al. Desmoid fibromatosis: a clinicopathologic study of 25 children. Semin Diagn Pathol 1986; 3: 138-150.

11. Faulkner LB, Hajdu SI, Kher U, et al. Pediatric desmoid tumor: retrospective analysis of 63 cases. J Clin Oncol 1995; 13: 2813-2818.

12. Buitendijk S, van de Ven CP, Dumans TG, et al. Pediatric aggressive fibromatosis: a retrospective analysis of 13 patients and review of literature. Cancer 2005; 104: 1090-1099.

13. Liu Q, Fang L, Li B. Desmoid fibromatosis in the foot: A case report and literature review. Medicine (Baltimore) 2018; 97; e13109.

14. Kabiri EH, Al Aziz S, El Maslout H, Benosman A. Desmoid tumors of the chest wall. Eur J Cardiothorac Surg 2001; 19: 580-583.

15. Mátrai Z, Tóth L, Szentirmay Z, et al. Sporadic desmoid tumors of the chest: long-term followup of 28 multimodally treated patients. Eur J Cardiothorac Surg 2011; 40: 1170-1176.

16. Kwee RM, Kwee TC. Calcified or ossified benign soft tissue lesions that may simulate malignancy. Skeletal Radiol 2019; 48: 1875-1890.

17. Allen PJ, Shriver CD. Desmoid tumors of the chest wall. Semin Thorac Cardiovasc Surg 1999; 11: 264-269.

18. Sivanesan E, Gitlin MC. Desmoid Tumors: A Review of the Literature and Pharmacologic Management. J Pain Palliat Care Pharmacother 2016; 30: 99-105.

19. Spear MA, Jennings LC, Mankin HJ, et al. Individualizing management of aggressive fibromatoses. Int J Radiat Oncol Biol Phys 1998; 40: 637-645.

20. Jabbari S, Andolino D, Weinberg V, et al. Successful treatment of high risk and recurrent pediatric desmoids using radiation as a component of multimodality therapy. Int J Radiat Oncol Biol Phys 2009; 75: 177-182.

21. Gronchi A, Colombo C, Le Péchoux C, et al. Sporadic desmoid-type fibromatosis: a stepwise approach to a non-metastasising neoplasm - a position paper from the Italian and the French sarcoma group. Ann Oncol 2013; 25: 578-583. 